\title{
Offline Trace Checking of Quantitative Properties of Service-Based Applications
}

\author{
Domenico Bianculli \\ SnT Centre - University of Luxembourg, Luxembourg \\ domenico.bianculli@uni.lu \\ Carlo Ghezzi, Srđan Krstić, Pierluigi San Pietro \\ DEEP-SE group - DEIB - Politecnico di Milano, Italy \\ \{carlo.ghezzi,srdan.krstic,pierluigi.sanpietro\}@ polimi.it
}

August 18, 2018

\begin{abstract}
Service-based applications are often developed as compositions of partner services. A service integrator needs precise methods to specify the quality attributes expected by each partner service, as well as effective techniques to verify these attributes. In previous work, we identified the most common specification patterns related to provisioning service-based applications and developed an expressive specification language (SOLOIST) that supports them. SOLOIST is an extension of metric temporal logic with aggregate temporal modalities that can be used to write quantitative temporal properties.

In this paper we address the problem of performing offline checking of service execution traces against quantitative requirements specifications written in SOLOIST. We present a translation of SOLOIST into $\operatorname{CLTLB}(\mathscr{D})$, a variant of linear temporal logic, and reduce the trace checking of SOLOIST to bounded satisfiability checking of CLTLB $(\mathscr{D})$, which is supported by ZOT, an SMT-based verification toolkit. We detail the results of applying the proposed offline trace checking procedure to different types of traces, and compare its performance with previous work.
\end{abstract}

\section{Introduction}

Service-based applications (SBAs) are one of the main approaches followed nowadays to develop modern enterprise information systems, adopting the paradigm of service-oriented computing [19]. SBAs are usually defined as service compositions, created by orchestrating several existing services, possibly provided by third-parties, by means of dedicated languages such as BPEL. Developing and operating an SBA involves many stakeholders: service endusers, the developers and providers of services used in the SBA, as well as the service integrators that realize the composite services. However, service integrators have the ultimate responsibility for maintaining an adequate level of quality attributes (e.g., in terms of functional correctness and QoS, quality of service) of the composite services they provide, independently of (but at the same time, based on) the guarantees and the service-level agreements offered by the providers of the services they compose. This can be achieved in a systematic and formal way by developing a specification language that can capture useful properties of SBAs and by providing means for verifying SBAs against properties written in such a specification language. 
Several verification techniques have been developed and tailored [10, 27, 12, 2] for the domain of SBAs, to assist service integrators in verification activities both at design time (e.g., testing, model checking) and run time (e.g., monitoring). In the case of formal approaches, the verification techniques adopt a temporal logic (such as LTL, CTL) as the specification language of the properties of interest. In the domain of composite SBAs, these properties express constraints on the interactions of the composite service with its partner services. In a previous work some of the authors developed SOLOIST (SpecificatiOn Language fOr servIce compoSitions inTeractions) [9] a metric temporal logic with new, additional temporal modalities that can express properties of SBAs in terms of bounds on some aggregated values, calculated over a certain time window. These modalities have been defined based on an extensive field study [8] of the requirements specifications in the context of service-based applications, and they are tailored to express the most common requirements occurring in practice. The study — performed in collaboration with an industrial partner - analyzed more than 900 requirements specifications, extracted both from research papers and industrial data, and led to the identification of a new class of specification patterns, specific to the domain of service provisioning. Examples of these patterns are those characterizing the average response time of a service invocation and the count/average/maximum number of event occurrences in a given time window.

In this paper we focus on the problem of performing offline checking of execution traces against requirements specifications written in SOLOIST. Trace checking (also called trace validation [24] or history checking [17]) is a procedure for evaluating a formal specification over a $\log$ of recorded events produced by a system, i.e., over a temporal evolution of the system. We assume that a trace is finite and composed by the events corresponding to the interactions of a composite service with its external services (e.g., invoking external service operations or receiving service requests). Traces can be produced by a proper monitoring/logging infrastructure, and made available at the end of the execution to perform offline trace checking.

The main contribution of the paper is an offline trace checking procedure for SOLOIST properties exploiting a translation into $\operatorname{CLTLB}(\mathscr{D})$ [6], an extension of PLTLB (Propositional Linear Temporal Logic with both past and future modalities) augmented with atomic formulae built over a constraint system $\mathscr{D}$. We chose $\operatorname{CLTLB}(\mathscr{D})$ as the target of our translation since it supports the definition of arithmetical constraints over a set of integer variables (also called counters); as we will detail in Sect. 3, these counters allow a compact and easy-toverify translation. We express the problem of trace checking of SOLOIST properties in terms of bounded satisfiability checking (BSC) of $\operatorname{CLTLB}(\mathscr{D})$ and rely on the BSC procedure for metric temporal logic [26] implemented in ZOT. We focus on requirements containing quantitative properties involving aggregate operations on events occurring in a given time window, like the average response time of a certain operation provided by a partner service.

In the original definition of SOLOIST [9] we showed how, under certain assumptions, the language can be translated into LTL, guaranteeing its decidability based on well-known results in temporal logic. However, this translation was only a proof of concept and was not meant to guarantee efficiency if one would use LTL-based verification procedures. In previous work [7] we introduced a trace checking procedure, based on another encoding of SOLOIST properties into formulae of QF-EUFIDL, the theory of quantifier-free integer difference logic with uninterpreted function and predicate symbols. This encoding was tailored for sparse traces, i.e., traces in which the number of time instants when events occur is very low with respect to the length of the trace. In contrast, the new encoding proposed in this paper supports a much more efficient checking of dense traces. In this paper we also compare the two approaches on traces of different degrees of sparseness.

The rest of the paper is organized as follows. Section 2 provides background information 
on SOLOIST and CLTLB $(\mathscr{D})$. We present the translation of SOLOIST into CLTLB $(\mathscr{D})$ in Section 3 . In section 4 we discuss some implementation details. Section 5 reports on the evaluation performed to assess the scalability of our approach, also in comparison with previous work. Section 6] surveys related work, and Sect. 7]concludes the paper, giving some directions for future work.

\section{Preliminaries}

\subsection{SOLOIST in a Nutshell}

Previous work [8] reports the results of a field study on the the specification patterns used to express requirements of SBAs. After analyzing more than 900 requirements specifications extracted both from research papers and industrial data, the authors of the study identified a set of specification patterns specific to service provisioning (in addition to the well-known ones like those defined in [20, 16]). The service provisioning patterns refer to: S1) average response time; S2) counting the number of events; S3) average number of events; S4) maximum number of events; S5) absolute time; S6) unbounded elapsed time; S7) data-awareness. On the basis of these findings, a new specification language, called SOLOIST (SpecificatiOn Language fOr servIce compoSitions inTeractions), was introduced in [9]. SOLOIST was in fact designed with the goal of supporting the common specification patterns found for service provisioning; it is a propositional metric temporal logic with new temporal modalities that support aggregate operations on events occurring in a given time window.

The syntax of SOLOIST is defined by the following grammar:

$\phi::=p|\neg \phi| \phi \wedge \phi\left|\phi \bigcup_{I} \phi\right| \phi \mathrm{S}_{I} \phi\left|\mathfrak{C}_{\bowtie n}^{K}(\phi)\right| \mathfrak{U}_{\bowtie \Perp n}^{K, h}(\phi)\left|\mathfrak{M}_{\bowtie n}^{K, h}(\phi)\right| \mathfrak{D}_{\bowtie n}^{K}(\phi, \phi)$

where $p \in \Pi$, with $\Pi$ being a finite set of atoms; $I$ is a nonempty interval over $\mathbb{N} ; n, K, h$ range over $\mathbb{N} ; \bowtie \in\{<, \leq, \geq\rangle,,=\}$. The arguments $\phi$ of modalities $\mathfrak{C}, \mathfrak{U}, \mathfrak{M}, \mathfrak{D}$ are restricted to atoms in $\Pi$. Moreover, the two arguments in the $\mathfrak{D}$ modality are required to be different atoms.

The $\mathrm{U}_{I}$ and $\mathrm{S}_{I}$ modalities are, respectively, the metric "Until" and "Since" operators. Additional temporal modalities can be derived using the usual conventions; for example "Always" is defined as $\mathrm{G}_{I} \phi \equiv \neg\left(\top \mathrm{U}_{I} \neg \phi\right)$ and "Eventually in the Past" as $\mathrm{P}_{I} \phi \equiv \top \mathrm{S}_{I} \phi$, where $\top$ means "true". The remaining modalities are called aggregate modalities. The $\mathfrak{C}_{\triangleright \bowtie n}^{K}(\phi)$ modality states a bound (represented by $\bowtie n$ ) on the number of occurrences of an event $\phi$ in the previous $K$ time instants; it expresses pattern $\mathrm{S} 2$. The $\mathfrak{U}_{\bowtie{ }^{\prime} n}^{K, h}(\phi)$ (respectively, $\mathfrak{M}_{\bowtie n}^{K, h}(\phi)$ ) modality expresses a bound on the average (respectively, maximum) number of occurrences of an event $\phi$, aggregated over the set of right-aligned adjacent non-overlapping subintervals within a time window $K$; it corresponds to pattern S3 (respectively, S4). A subtle difference in the semantics of the $\mathfrak{U}$ and $\mathfrak{M}$ modalities is that $\mathfrak{M}$ considers events in the (possibly empty) tail interval, i.e., the leftmost observation subinterval whose length is less than $h$, while the $\mathfrak{U}$ modality ignores them. The $\mathfrak{D}_{\bowtie n}^{K}(\phi, \psi)$ modality expresses a bound on the average time elapsed between a pair of specific adjacent events $\phi$ and $\psi$ occurring in the previous $K$ time instants; it can be used to express pattern S1.

The formal semantics of SOLOIST is trace-based, i.e., defined on timed $\omega$-words over $2^{\Pi} \times \mathbb{N}$. A timed sequence $\tau=\tau_{0} \tau_{1} \ldots$ is an infinite sequence of values $\tau_{i} \in \mathbb{N}$ satisfying $\tau_{i}<\tau_{i+1}$, for all $i \geq 0$, i.e., the sequence increases strictly monotonically. A timed $\omega$-word over alphabet $2^{\Pi}$ is a pair $(\sigma, \tau)$ where $\sigma=\sigma_{0} \sigma_{1} \ldots$ is an infinite word over $2^{\Pi}$ and $\tau$ is a timed sequence. A timed language over $2^{\Pi}$ is a set of timed words over the same alphabet. Notice that there is a distinction between the integer position $i$ in the timed $\omega$-word and the corresponding integer timestamp $\tau_{i}$. Figure 1 defines the satisfiability relation $(w, i) \models \phi$ for 


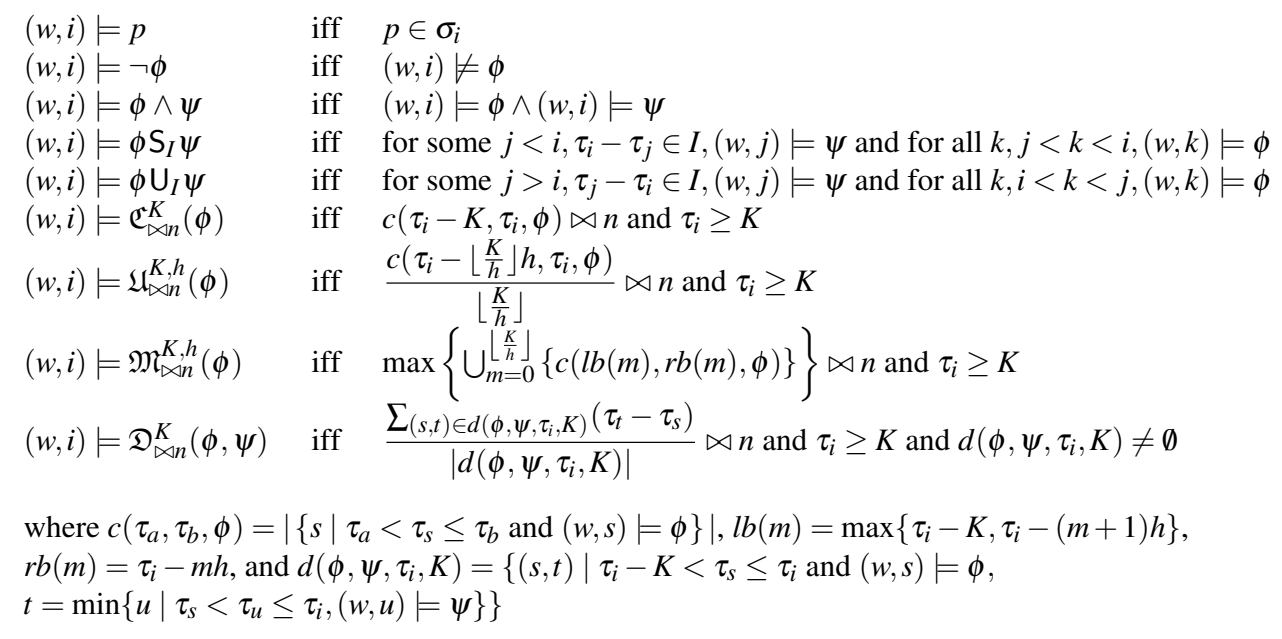

Figure 1: Formal semantics of SOLOIST

every timed $\omega$-word $w$, every position $i \geq 0$ and for every SOLOIST formula $\phi$.

We remark that the version of SOLOIST presented here is a restriction of the original one in [9]. To simplify the presentation in the next sections, we dropped first-order quantification on finite domains (which was introduced to support data-awareness, i.e., pattern S7 ) and limited the argument of the $\mathfrak{D}$ modality to only one pair of events. These restrictions are only syntactic sugar and we refer to [9] for the details of the transformations that provide support for them.

\subsection{Example}

In this section we show how to express common quantitative properties of an SBA by means of SOLOIST. As an example, we consider an SBA realized as a service composition described in BPEL, depicted in Fig. 2] using the (visually intuitive) notation introduced in [1].

The process ATMFrontEnd starts when the receive activity logOn processes a message from the SessionManager service. This starts a customer session: the process verifies whether the customer holds a valid account at the bank, by invoking the checkAccess operation of the BankAccount service. If the latter identifies the customer, a loop is started to manage the customer's requests sent via the UserInteraction service. The customerMenu pick activity, contained in the body of the loop, may receive four kinds of possible requests: three of them (getBalance, deposit, withdraw) are forwarded to the corresponding operations of the BankAccount service; the logOff request terminates the loop, closing the customer session.

To annotate a BPEL process with SOLOIST, we denote the execution of each activity with an atom. For example, receive activity $\log 0 \mathrm{n}$ can be denoted with an atom $\log O n \in \Pi$. Synchronous invoke activities are actually modeled with two atoms, corresponding to the start and the end of the invocation; these are denoted with the "_start" and "_end" suffixes, respectively.

Below we list some examples of quantitative properties expressed first in natural language and then with SOLOIST; more details on the features of the language are available in [9]. All properties are under the scope of an implicit universal temporal quantification as in "In every process run, ..."; we assume the time units to be in seconds. 


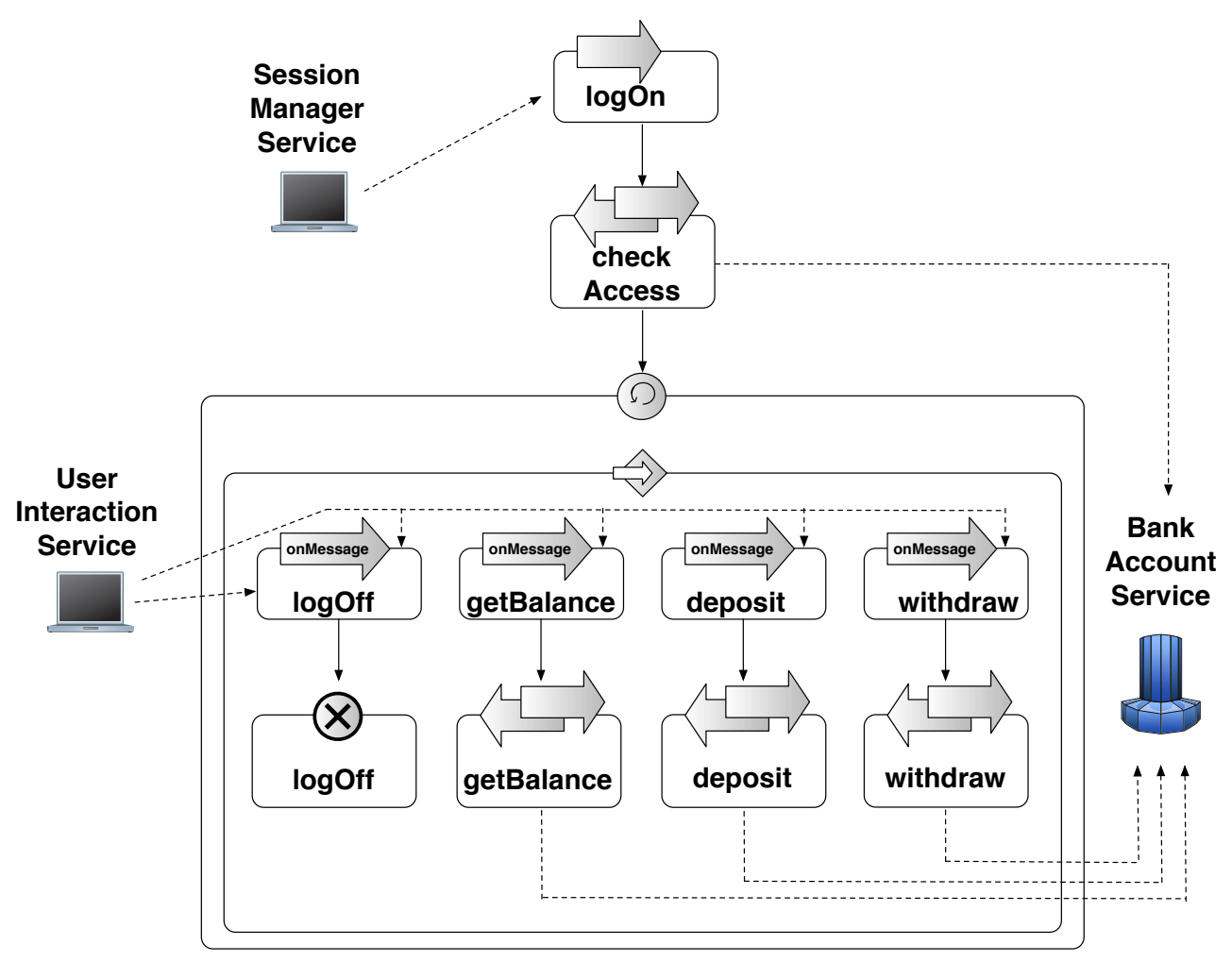

Figure 2: ATMFrontEnd business process

QP1: WithdrawalLimit

The number of withdrawal operations performed within 10 minutes before customer $\operatorname{logs}$ off is less than or equal to the allowed limit (assumed to be 3, for example). This property is expressed as:

$\mathrm{G}\left(\log O f f \rightarrow \mathfrak{C}_{\leq 3}^{600}(\right.$ withdraw $\left.)\right)$.

QP2: CheckAccessAverageResponseTime

The average response time of operation checkAccess provided by the BankAccount service is always less than 5 seconds within any 15 minute time window. This property is expressed as:

$\mathrm{G}\left(\mathfrak{D}_{<5}^{900}\{(\right.$ checkAccess_start, checkAccess_end $\left.)\}\right)$.

QP3: MaxNumberOfBalancelnquiries

The maximum number of balance inquiries is restricted to at most 2 per minute within 10 minutes before customer session ends. This property is expressed as:

$\mathrm{G}\left(\log O f f \rightarrow \mathfrak{M}_{\leq 2}^{600,60}(\right.$ getBalance $\left.)\right)$.

\section{$2.3 \operatorname{CLTLB}(\mathscr{D})$}

$\operatorname{CLTLB}(\mathscr{D})[6]$ is an extension of PLTLB (Propositional Linear Temporal Logic with both past and future modalities) [23] augmented with atomic formulae built over a constraint system $\mathscr{D}$. In practice, $\operatorname{CLTLB}(\mathscr{D})$ defines a set of variables $C$ and arithmetical constraints over a constraint system $\mathscr{D}$; in our case, $\mathscr{D}$ is the structure $\left(\mathbb{Z},=,\left(<_{d}\right)_{d \in \mathbb{Z}}\right)$. For this particular 
combination, decidability of $\operatorname{CLTLB}(\mathscr{D})$ has been proven in [15]. Each $<_{d}$ is a binary relation defined as $x<_{d} y \Leftrightarrow x<y+d$, hence, for example, the notation $x=y+d$ is an abbreviation for $y<_{1-d} x \wedge x<_{d+1} y$. Variables (henceforth called counters) receive a separate evaluation at each time instant. In addition to the standard PLTLB temporal operators "Since" and "Until", $\operatorname{CLTLB}(\mathscr{D})$ introduces the new construct of arithmetic temporal term, defined as $\alpha:=c|x|$ $\mathrm{Y}(x) \mid \mathrm{X}(x)$, where $c \in \mathbb{Z}$ is a constant, $x \in C$ is a counter and $\mathrm{Y}$ and $\mathrm{X}$ are temporal operators applied to counters. These temporal operators for counters return the value of the counter in the previous and in the next time instant, respectively. Note that we use a syntactically sugared version of PLTLB using metric temporal operators over time intervals, such as $\mathrm{U}_{I}$. Since time is discrete, they are just a convenient shorthand [25]. The syntax of CLTLB( $\mathscr{D})$ is the following:

$\phi::=p|\alpha \sim \alpha| \neg \phi|\phi \wedge \phi| \phi \mathrm{U}_{I} \phi\left|\phi \mathrm{S}_{I} \phi\right| \mathrm{X} \phi \mid \mathrm{Y} \phi$

where $p$ is an atomic proposition, $\sim \in\left\{=,\left(<_{d}\right)_{d \in \mathbb{Z}}\right\}, \mathrm{S}_{I}, \mathrm{U}_{I}, \mathrm{X}, \mathrm{Y}$ are the usual "Since", "Until", "Next", and "Yesterday" modalities of PLTLB. Additional temporal modalities (like G, "Globally", and W, "Weak Until") can be defined using the usual conventions. An example of a $\operatorname{CLTLB}(\mathscr{D})$ formula is $\mathrm{G}(\phi \rightarrow \mathrm{X}(y)=y+1)$, which states that whenever $\phi$ is true, the value of counter $y$ in the next time instant must be incremented of 1 with respect to the value at the current time instant.

$\operatorname{CLTLB}(\mathscr{D})$ formulae admit finite, ultimately periodic two-part models $(\pi, \delta)$. Function $\pi: \mathbb{N} \rightarrow \mathscr{P}(\Pi)$ associates a subset of the propositions with each time instant, while function $\delta: \mathbb{N} \times C \rightarrow \mathbb{Z}$ defines the value of counters at each time position. Hereafter, this two-part model will be graphically represented as in Fig. 5. the topmost row (above the timeline) represents function $\pi$ (e.g., $\pi(5)=\{\psi\}$ ); the rows of integers below the timeline represent function $\delta$, i.e., the values of each counter defined in the model. In the example in the figure there are six counters, as shown on the left: $c_{\chi}, g_{\phi, \psi}, h_{\phi, \psi}, s_{\phi, \psi}, a_{\phi, \psi}, b_{\phi, \psi}$; the $\delta$ function is defined so that we have, for example in correspondence with the sixth time instant (position $\# 5), \delta\left(5, g_{\phi, \psi}\right)=1, \delta\left(5, h_{\phi, \psi}\right)=0, \delta\left(5, s_{\phi, \psi}\right)=3, \delta\left(5, a_{\phi, \psi}\right)=0$, and $\delta\left(5, b_{\phi, \psi}\right)=3$.

\section{The Translation from SOLOIST to $\operatorname{CLTLB}(\mathscr{D})$}

The key point in defining the translation from SOLOIST to CLTLB $(\mathscr{D})$ is to bridge the gap between the semantics of SOLOIST based on timed $\omega$-words, where the temporal information is denoted by an integer time-stamp, and the one of CLTLB $(\mathscr{D})$, where the temporal information is implicitly defined by the integer position in an $\omega$-word. The two temporal models can be transformed into each other. Here we are interested in pinpointing, in a $\operatorname{CLTLB}(\mathscr{D}) \omega$-word, only the positions that correspond to actual time-stamps in a SOLOIST timed $\omega$-word. These timestamps correspond to instants where some event actually occurs. To do so, we add to the set $\Pi$ a special propositional symbol $e$, which is true in each position corresponding to a "valid" time-stamp in the timed $\omega$-word; a "valid" time-stamp is one where at least an event, represented by a propositional symbol, occurs. An example of this conversion is shown in Fig. 3. where a timed $\omega$-word is depicted in the timeline at the top and its equivalent $\omega$-word corresponds to the timeline at the bottom; notice the special symbols $\neg e$ that hold in positions in the $\omega$-word which do not correspond to a "valid" time-stamp in the timed $\omega$-word. Hereafter, when displaying $\omega$-words, we will omit the symbol $e$ from positions in the timeline, since its presence can be implied by the presence of other propositional symbols in the same position in the timeline.

To define the translation from SOLOIST to $\operatorname{CLTLB}(\mathscr{D})$ we consider, without loss of expressiveness, only formulae in positive normal form, i.e., where negation may only occur on atoms (see, for example, [25]). First, we extend the syntax of the language by introducing 


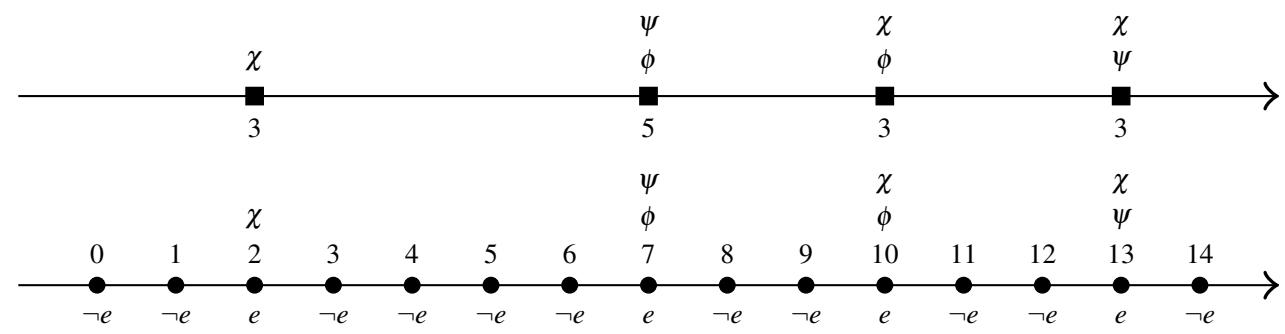

Figure 3: Mapping a timed $\omega$-word into an $\omega$-word

a dual version for each operator in the original syntax, except for the $\mathfrak{C}_{\bowtie n}^{K}, \mathfrak{U}_{\bowtie \Perp n}^{K, h}, \mathfrak{M}_{\bowtie \bowtie n}^{K, h}, \mathfrak{D}_{\bowtie n}^{K}$ modalities 1 the dual of $\wedge$ is $\vee$; the dual of $\mathrm{U}_{I}$ is "Release" $\mathrm{R}_{I}: \phi \mathrm{R}_{I} \psi \equiv \neg\left(\neg \phi \mathrm{U}_{I} \neg \psi\right)$; the dual of $\mathrm{S}_{I}$ is "Trigger" $\mathrm{T}_{I}: \phi \mathrm{T}_{I} \psi \equiv \neg\left(\neg \phi \mathrm{S}_{I} \neg \psi\right)$. A formula is in positive normal form if its alphabet is $\left\{\wedge, \vee, \cup_{I}, \mathrm{R}_{I}, \mathrm{~S}_{I}, \mathrm{~T}_{I}, \mathfrak{C}_{\bowtie n}^{K}, \mathfrak{U}_{\bowtie n}^{K, h}, \mathfrak{M}_{\bowtie n}^{K, h}, \mathfrak{D}_{\bowtie n}^{K}\right\} \cup \Pi \cup \bar{\Pi}$, where $\bar{\Pi}$ is the set of formulae of the form $\neg p$ for $p \in \Pi$.

We can now illustrate the translation $\rho$ from SOLOIST formulae to CLTLB $(\mathscr{D})$. For the propositional $\left(\neg, \wedge\right.$ and $\vee$ ) and temporal part $\left(\mathrm{U}_{I}, \mathrm{~S}_{I}, \mathrm{R}_{I}\right.$ and $\left.\mathrm{T}_{I}\right)$ of SOLOIST the translation is straightforward:

$$
\begin{aligned}
\rho(p) & \equiv p, p \in \Pi \\
\rho(\neg p) & \equiv \neg p, p \in \Pi \\
\rho(\phi \wedge \psi) & \equiv \rho(\phi) \wedge \rho(\psi) \\
\rho(\phi \vee \psi) & \equiv \rho(\phi) \vee \rho(\psi) \\
\rho\left(\phi \mathrm{U}_{I} \psi\right) & \equiv(\neg e \vee \rho(\phi)) \mathrm{U}_{I}(e \wedge \rho(\psi)) \\
\rho\left(\phi \mathrm{S}_{I} \psi\right) & \equiv(\neg e \vee \rho(\phi)) \mathrm{S}_{I}(e \wedge \rho(\psi)) \\
\rho\left(\phi \mathrm{R}_{I} \psi\right) & \equiv(e \wedge \rho(\phi)) \mathrm{R}_{I}(\neg e \vee \rho(\psi)) \\
\rho\left(\phi \mathrm{T}_{I} \psi\right) & \equiv(e \wedge \rho(\phi)) \mathrm{T}_{I}(\neg e \vee \rho(\psi))
\end{aligned}
$$

In the rest of this section we focus on the translation of the $\mathfrak{C}_{\bowtie \Perp}^{K}, \mathfrak{U}_{\bowtie n}^{K, h}, \mathfrak{M}_{\bowtie n}^{K, h}$ and $\mathfrak{D}_{\bowtie n}^{K}$ modalities.

\subsection{Translation of the $\mathfrak{C}$ modality}

The $\mathfrak{C}$ modality expresses a bound on the number of occurrences of a certain event in a given time window; it comes natural to use the counters available in $\operatorname{CLTLB}(\mathscr{D})$ for the translation. Indeed, for each sub-formula of the form $\mathfrak{C}_{\triangleright \bowtie n}^{K}(\chi)$, we introduce a counter $c_{\chi}$, constrained by a set of $\operatorname{CLTLB}(\mathscr{D})$ axioms, detailed below. Informally, these axioms define the value of $c_{\chi}$ such that at each time position it captures the number of occurrences of event $\chi$ seen in the past:
A1) $c_{\chi}=0$
A2) $\mathrm{G}\left((e \wedge \chi) \rightarrow \mathrm{X}\left(c_{\chi}\right)=c_{\chi}+1\right)$
A3) $\mathrm{G}\left((\neg e \vee \neg \chi) \rightarrow \mathrm{X}\left(c_{\chi}\right)=c_{\chi}\right)$

\footnotetext{
${ }^{1}$ A negation in front of one of the $\mathfrak{C}_{\bowtie \bowtie n}^{K}, \mathfrak{U}_{\bowtie \rtimes n}^{K, h}, \mathfrak{M}_{\bowtie \text { }}^{K, h}, \mathfrak{D}_{\bowtie n}^{K}$ modalities becomes a negation of the relation denoted by the $\bowtie$ symbol, hence no dual version is needed for them.
} 


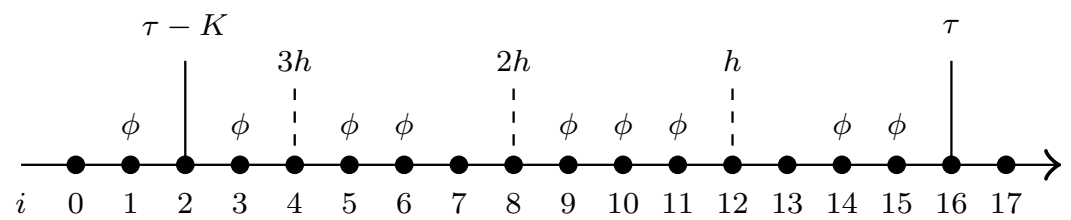

Figure 4: Sample trace showing the time window and the observation subintervals considered for the evaluation of the $\mathfrak{M}_{\geq 1}^{14,4}(\phi)$ formula at time instant $\tau=16$

Axiom $\mathrm{A} 1$ initializes the counter to zero. Axiom $\mathrm{A} 2$ states that if there is an occurrence of a valid event $\chi$, (denoted by $e \wedge \chi$ ) the value of the counter $c_{\chi}$ in the next time instant is increased by one with respect to the value at the current time instant. Axiom $A 3$ refers to the opposite situation, when either there is no occurrence of the event $\chi$ or the time instant is not valid (i.e., $e$ does not hold in that time instant). In this case, the value of the counter in the next time instant must have the same value as in the current time instant. Both axioms $\mathrm{A} 2$ and A 3 have to hold at every time instant, so they are in the scope of a globally temporal operator.

We can calculate the exact number of occurrences by subtracting the values of the counter at the appropriate time instants; we explain this through the example in Fig. 5, which depicts a short trace of length 21 and the values assumed by the counter $c_{\chi}$ (in the first row) at each time instant, as determined by the axioms. In the example, to evaluate the formula $\mathfrak{C}_{>1}^{K}(\chi)$ with $K=11$ at time instant $t=16$, we subtract from the value of the counter $c_{\chi}$ at time instant $t+1=17$ (since we want to consider a possible occurrence of $\chi$ at time instant $t$ ) the value of the counter at time instant 6 (i.e., $t-(K-1)=16-(11-1)$, which is 11 time instants in the past with respect to time instant $t+1)$; these values are enclosed in the figure with diamond markers. The value resulting from the subtraction $6-1=5$ is then compared to the specified bound $(5>1)$. In symbols, this can be written as $\mathrm{X}\left(c_{\chi}\right)-\mathrm{Y}^{10}\left(c_{\chi}\right)>1$ evaluated at time instant $t$. This intuition is captured by the following $\operatorname{CLTLB}(\mathscr{D})$ formula, which generalizes the translation of a SOLOIST sub-formula of the form $\mathfrak{C}_{\bowtie n}^{K}(\chi)$ :

$$
\rho\left(\mathfrak{C}_{\bowtie n}^{K}(\chi)\right) \equiv \mathrm{X}\left(c_{\chi}\right)-\mathrm{Y}^{K-1}\left(c_{\chi}\right) \bowtie n
$$

Notice that the axioms are conjuncted with the resulting translation of the SOLOIST formula, thus effectively constraining the behavior of all the counters of type $c_{\chi}$.

\subsection{Translation of the $\mathfrak{U}$ modality}

The translation of the $\mathfrak{U}$ modality is defined in terms of the $\mathfrak{C}$ modality; it can then be defined as follows:

$$
\rho\left(\mathfrak{U}_{\bowtie n}^{K, h}(\phi)\right) \equiv \rho\left(\mathfrak{C}_{\bowtie n \cdot\left\lfloor\frac{K}{h}\right\rfloor}^{\left\lfloor\frac{K}{h}\right\rfloor \cdot h}(\phi)\right)
$$

This translation ignores the tail subinterval of the $\mathfrak{U}$ modality, which is consistent with the SOLOIST semantics [9]. 


\subsection{Translation of the $\mathfrak{M}$ modality}

To translate the $\mathfrak{M}$ modality we rely on the $\mathfrak{C}$ modality. The translation of a formula of the form $\mathfrak{M}_{<n}^{K, h}(\phi)$ is defined as: $\rho\left(\mathfrak{M}_{<n}^{K, h}(\phi)\right) \equiv$

$$
\left(\bigwedge_{m=0}^{\left\lfloor\frac{K}{h}\right\rfloor-1} \mathrm{Y}^{m \cdot h}\left(\rho\left(\mathfrak{C}_{<n}^{h} \phi\right)\right)\right) \wedge\left(\mathrm{Y}^{\left\lfloor\frac{K}{h}\right\rfloor \cdot h}\left(\rho\left(\mathfrak{C}_{<n}^{(K \bmod h)} \phi\right)\right)\right)
$$

For a formula of the form $\mathfrak{M}_{>n}^{K, h}(\phi)$ we have: $\rho\left(\mathfrak{M}_{>n}^{K, h}(\phi)\right) \equiv$

$$
\left(\bigvee_{m=0}^{\left\lfloor\frac{K}{h}\right\rfloor-1} \mathrm{Y}^{m \cdot h}\left(\rho\left(\mathfrak{C}_{>n}^{h} \phi\right)\right)\right) \vee\left(\mathrm{Y}^{\left\lfloor\frac{K}{h}\right\rfloor \cdot h}\left(\rho\left(\mathfrak{C}_{>n}^{(K \bmod h)} \phi\right)\right)\right)
$$

The formula decomposes the computation of the maximum number of occurrences of the event $(e \wedge \phi)$ by suitably combining constraints on the number of occurrences of the event in each observation interval $h$ within the time window $K$. The other cases of the operator $\bowtie$ can be defined in a similar way.

Fig. 4 shows an example trace of length 18 . We evaluate the formula $\mathfrak{M}_{\geq 3}^{14,4}(\phi)$ at time instant $\tau=16$. The vertical solid lines delimit the time window of length $K=14$; the dashed lines delimit the adjacent non-overlapping observation subintervals of length $h=4$. The $\mathfrak{M}$ modality formula is translated into a disjunction of four $\mathfrak{C}$ modality formulae each referring to a different subinterval. The first three $\left(\left\lfloor\frac{K}{h}\right\rfloor=\left\lfloor\frac{14}{4}\right\rfloor=3\right)$ formulae have the form $\mathfrak{C}_{\geq 3}^{4}(\phi)$ and are evaluated at time instants $16(=16-0 \cdot 4), 12(=16-1 \cdot 4)$ and $8(=16-2 \cdot 4)$. The fourth formula (corresponding to rightmost disjunct defined in the translation $\rho$ ) has the form $\mathfrak{C}_{\geq 3}^{2}(\phi)$ and is evaluated at time instant $4\left(=16-\left\lfloor\frac{14}{4}\right\rfloor \cdot 4\right)$. We can conclude that, the formula $\mathfrak{M}_{\geq 3}^{14,4}(\phi)$ holds at time instant $\tau=16$ since formula $\mathfrak{C}_{\geq 3}^{4}(\phi)$ holds at time instant 12 and renders the disjunction true.

\subsection{Translation of the $\mathfrak{D}$ modality}

The $\mathfrak{D}$ modality expresses a bound on the average distance between the occurrences of pairs of events in a given time window. As anticipated in Sect. 2.1, we consider only (sub)formulae of the $\mathfrak{D}$ modality that refer to one pair, like $\mathfrak{D}_{\bowtie n}^{K}(\phi, \psi)$.

Events, corresponding to atomic propositions in SOLOIST, can occur multiple times in a trace; when we refer to a specific occurrence of an event $\phi$ at a time instant $\tau$, we denote this as $\phi_{\mid \tau}$. Clearly, a pair of events $(\phi, \psi)$ may also have multiple instances in a trace. We call a pair of the form $\left(\phi_{\mid i}, \psi_{\mid j}\right)$ an instance if there is an occurrence of event $\phi$ at time instant $i$ and an occurrence of event $\psi$ at time instant $j$, with $i<j$. We call such instance open at time instant $\tau$ if $i \leq \tau<j$. Otherwise, the instance is closed at time instant $\tau$. The distance of a closed (pair) instance is $j-i$; for an open pair at time instant $\tau$, the distance is $\tau-i$. A time window of length $K$ defined for a $\mathfrak{D}$ modality (sub-)formula evaluated at time instant $\tau$ is bounded by the time instants $\tau+1$ and $\tau-K+1$. For a certain trace, we say that a $\mathfrak{D}$ modality (sub-)formula for a pair of events $(\phi, \psi)$ has a left-open pair in the trace if there is an open instance of $(\phi, \psi)$ at time instant $\tau-K+1$ in the trace; similarly, we say that the (sub-)formula has a right-open pair in the trace if there is an open instance of $(\phi, \psi)$ at time instant $\tau+1$ in the trace. The translation has then to take into account four distinct cases, depending on whether a $\mathfrak{D}$ modality (sub-)formula contains either (left- and/ or right-) open pairs or none. 
As done in the case of the $\mathfrak{C}$ modality, the translation is based on CLTLB $(\mathscr{D})$ counters. For each sub-formula of the form $\mathfrak{D}_{\bowtie n}^{K}(\phi, \psi)$, we introduce five counters, namely:

- $g_{\phi, \psi}$ : this binary counter assumes value 1 in the time instants following an occurrence of $\phi$ and it is reset to 0 after an occurrence of $\psi$. It acts as a flag denoting the time instants during which the event pair instance is open;

- $h_{\phi, \psi}$ : in each time instant, this counter contains the number of previously-seen closed pair instances. It is increased after every occurrence of $\psi$;

- $s_{\phi, \psi}$ : at each time instant, the value of this counter corresponds to the sum of distances of all previously occurred pair instances. It is increased at every time instant when either $g_{\phi, \psi}=1$ holds or $\phi$ occurs;

- $a_{\phi, \psi}$ : this counter keeps track of the sum of the distances of all previously occurred closed pair instances;

- $b_{\phi, \psi}$ : this counter has the values that will be assumed by counter $s_{\phi, \psi}$ at the next occurrence of $\psi$ (more details below).

Counters $a_{\phi, \psi}, b_{\phi, \psi}$, and $h_{\phi, \psi}$ are directly used in the translation of the $\mathfrak{D}$ modality (sub)formulae, while counters $g_{\phi, \psi}$ and $s_{\phi, \psi}$ are helper counters, used to determine the values of the other counters. These five counters are constrained by the following axioms:

A4) $g_{\phi, \psi}=0 \wedge h_{\phi, \psi}=0 \wedge a_{\phi, \psi}=0 \wedge s_{\phi, \psi}=0$

A5) $\left(\mathrm{X}\left(b_{\phi, \psi}\right)=b_{\phi, \psi}\right) \mathrm{W}(e \wedge \psi)$

A6) $\mathrm{G}\left((e \wedge \phi \wedge \neg \psi) \rightarrow\left(\mathrm{X}\left(g_{\phi, \psi}\right)=1 \wedge \mathrm{X}\left(s_{\phi, \psi}\right)=s_{\phi, \psi}+1 \wedge \mathrm{X}\left(h_{\phi, \psi}\right)=h_{\phi, \psi} \wedge \mathrm{X}\left(a_{\phi, \psi}\right)=\right.\right.$ $\left.\left.a_{\phi, \psi}\right)\right)$

A7) $\mathrm{G}\left((e \wedge \psi \wedge \neg \phi) \rightarrow\left(\mathrm{X}\left(g_{\phi, \psi}\right)=0 \wedge \mathrm{X}\left(h_{\phi, \psi}\right)=h_{\phi, \psi}+1 \wedge \mathrm{X}\left(a_{\phi, \psi}\right)=s_{\phi, \psi} \wedge \mathrm{X}\left(s_{\phi, \psi}\right)=\right.\right.$ $\left.\left.s_{\phi, \psi} \wedge b_{\phi, \psi}=s_{\phi, \psi} \wedge \mathrm{X}\left(\left(\mathrm{X}\left(b_{\phi, \psi}\right)=b_{\phi, \psi}\right) \mathrm{W}(e \wedge \psi)\right)\right)\right)$

A8) $\mathrm{G}\left((\neg e \vee(\neg \phi \wedge \neg \psi)) \rightarrow\left(\mathrm{X}\left(g_{\phi, \psi}\right)=g_{\phi, \psi} \wedge \mathrm{X}\left(h_{\phi, \psi}\right)=h_{\phi, \psi} \wedge \mathrm{X}\left(a_{\phi, \psi}\right)=a_{\phi, \psi} \wedge\left(g_{\phi, \psi}=\right.\right.\right.$ $\left.\left.\left.1 \rightarrow \mathrm{X}\left(s_{\phi, \psi}\right)=s_{\phi, \psi}+1\right) \wedge\left(g_{\phi, \psi}=0 \rightarrow \mathrm{X}\left(s_{\phi, \psi}\right)=s_{\phi, \psi}\right)\right)\right)$

A9) $\mathrm{G}\left((e \wedge \phi \wedge \psi) \rightarrow\left(\mathrm{X}\left(g_{\phi, \psi}\right)=g_{\phi, \psi} \wedge \mathrm{X}\left(h_{\phi, \psi}\right)=h_{\phi, \psi}+1 \wedge \mathrm{X}\left(a_{\phi, \psi}\right)=a_{\phi, \psi} \wedge \mathrm{X}\left(s_{\phi, \psi}\right)=\right.\right.$ $s_{\phi, \psi} \wedge \mathrm{X}\left(\left(\mathrm{X}\left(b_{\phi, \psi}\right)=b_{\phi, \psi}\right) \mathrm{W}(e \wedge \psi)\right)$

Axiom $A$ initializes all counters except counter $b_{\phi, \psi}$, which will assume values determined by counter $s_{\phi, \psi}$. Axiom $A 5$ states that the value of counter $b_{\phi, \psi}$ will stay the same in all the time instants until the first occurrence of $\psi$. Notice that we use the $\mathrm{W}$ modality ("weak until"), to deal with traces without occurrences of $\psi$. Axiom $A 6$ determines the next time instant value of the following counters, upon occurrence of a $\phi$ and absence of a $\psi$ event (denoted by $e \wedge \phi \wedge \neg \psi$ ): counter $g_{\phi, \psi}$ is set to 1 ; counter $s_{\phi, \psi}$ is incremented by 1 ; counters $h_{\phi, \psi}$ and $a_{\phi, \psi}$ are constrained not to change in the next time instant. Axiom A 7 determines how the counters are updated when a $\psi$ event occurs and a $\phi$ event does not: counter $g_{\phi, \psi}$ is set to 0 ; counters $b_{\phi, \psi}, \mathrm{X} a_{\phi, \psi}$, and $\mathrm{X} s_{\phi, \psi}$ are set to be equal to $s_{\phi, \psi}$. Moreover, a formula equivalent to axiom $A 5$ holds in the next time instant, forcing the value of $b_{\phi, \psi}$ to stay the same in all the following time instants until the next occurrence of $\psi$. Axiom $A 8$ covers the cases either when there are no valid events or when neither $\phi$ nor $\psi$ occur. In these cases the values of counters $g_{\phi, \psi}, h_{\phi, \psi}$, and $a_{\phi, \psi}$ are constrained to stay the same, while counter $b_{\phi, \psi}$ is unconstrained. As for counter $s_{\phi, \psi}$, we need to distinguish two separate cases: when the pair instance is open (denoted by $g_{\phi, \psi}=1$ ), counter $s_{\phi, \psi}$ is incremented by 1 , otherwise it stays the same. Axiom A 9 handles the case when both events $\phi$ and $\psi$ hold, by incrementing counter $h_{\phi, \psi}$ by 1 and constraining the value of counter $b_{\phi, \psi}$ in the same way like axiom $\mathrm{A} 7$. The values of the other counters are constrained to stay the same.

As said above, the $b_{\phi, \psi}$ counter keeps the values that will be assumed by counter $s_{\phi, \psi}$ at the next occurrence of $\psi$. The value assumed by both counters $a_{\phi, \psi}$ and $b_{\phi, \psi}$ originates from 


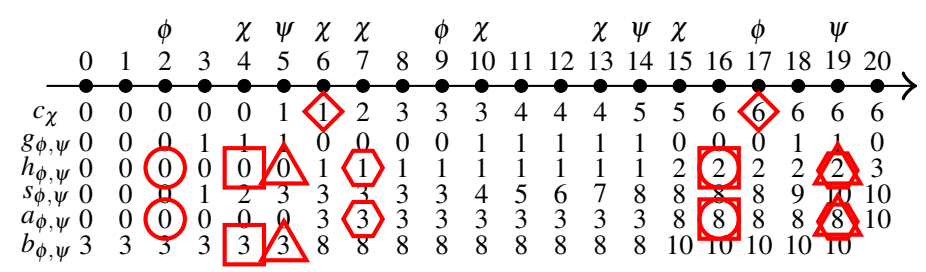

Figure 5: Sample trace showing the counters used for the translation of the $\mathfrak{C}$ and $\mathfrak{D}$ modalities

counter $s_{\phi, \psi}$, as enforced by axiom A 7 Axioms $A 6$ and $A 8$ make sure the value of $s_{\phi, \psi}$ is propagated in the future via counter $a_{\phi, \psi}$, while axiom $A 7$ enables the propagation of this value in the past via counter $b_{\phi, \psi}$. We elaborate this through an example: Fig. 5 represents a short trace with event $\psi$ occurring at time instants 5, 14, and 19. Axiom $A 5$ enforces equality between successive values of counter $b_{\phi, \psi}$ at adjacent time instants until the first occurrence of $\psi$ (time instants 0-5). Additional equalities (of the same type) on the values of counter $b_{\phi, \psi}$ are enforced by axiom A7 (time instants 6-14 and 15-19). The same axiom also determines equality between the values of the $s_{\phi, \psi}$ and $b_{\phi, \psi}$ counters upon an occurrence of $\psi$ (time instants 5, 14 and 19).

The translation $\rho\left(\mathfrak{D}_{\bowtie n}^{K}(\phi, \psi)\right)$ is defined as:

$$
\begin{aligned}
\text { if } 2\left(Y^{K-1}\left(g_{\phi, \psi}\right)=1\right) & \operatorname{then}\left(\frac{\mathrm{X}\left(a_{\phi, \psi}\right)-\mathrm{Y}^{K-1}\left(b_{\phi, \psi}\right)}{\mathrm{X}\left(h_{\phi, \psi}\right)-\mathrm{Y}^{K-1}\left(h_{\phi, \psi}\right)-1} \bowtie n \wedge Z_{1}\right) \\
& \operatorname{else}\left(\frac{\mathrm{X}\left(a_{\phi, \psi}\right)-\mathrm{Y}^{K-1}\left(a_{\phi, \psi}\right)}{\mathrm{X}\left(h_{\phi, \psi}\right)-\mathrm{Y}^{K-1}\left(h_{\phi, \psi}\right)} \bowtie n \wedge Z_{2}\right)
\end{aligned}
$$

The condition $\mathrm{Y}^{K}\left(g_{\phi, \psi}\right)=1$ checks whether the time window contains an open pair instance on its left bound. Since the semantics of the $\mathfrak{D}$ modality considers only closed pairs within the time window to compute the average distance, open pairs must be ignored both on the left and on the right bound of the time window. There is no need to differentiate between the cases when there is a right-open pair, since counter $a_{\phi, \psi}$ only considers distances between closed pair instances. The numerator of the fraction in both the then and else branches denotes the total distance, while the denominator corresponds to the number of pair instances considered for computing the total distance. Propositions $Z_{1}$ and $Z_{2}$ are respectively $\mathrm{X}\left(h_{\phi, \psi}\right)-\mathrm{Y}^{K-1}\left(h_{\phi, \psi}\right) \neq 1$ and $\mathrm{X}\left(h_{\phi, \psi}\right)-\mathrm{Y}^{K-1}\left(h_{\phi, \psi}\right) \neq 0$; due to these disjuncts the $\mathfrak{D}$ modality evaluates to true when there are no closed pairs in the time window $K$. Axioms $\mathrm{A} 4$. $A 5, A 6, A 7, A 8, A 9$ are conjuncted with the resulting translation and added as constraints that hold at the initial time instant of the trace.

An example of the use of counters to evaluate a formula with the $\mathfrak{D}$ modality is shown in Fig. 5. which depicts a simple trace and the values assumed by the counters $g_{\phi, \psi}, h_{\phi, \psi}, s_{\phi, \psi}$, $a_{\phi, \psi}$, and $b_{\phi, \psi}$ at each time instant, as determined by the axioms. We notice that there are three instances of the $(\phi, \psi)$ pair. If we evaluate the formula $\mathfrak{D}_{\bowtie n}^{14}(\phi, \psi)$ at time instant 15 , the two pair instances $\left(\phi_{\mid 2}, \psi_{\mid 5}\right)$ and $\left(\phi_{\mid 9}, \psi_{\mid 14}\right)$, considered to compute the average distance, are closed. The left-hand side (lhs) of the comparison operator $(\bowtie)$ is evaluated using the values of counters $a_{\phi, \psi}$ and $h_{\phi, \psi}$ at time instants 16 and 2 (enclosed in a circle in the figure), resulting in $\frac{8}{2}=4$. When the same formula is evaluated at time instant 18 , the portion of the trace considered contains both a left-open $\left(\phi_{\mid 2}, \psi_{\mid 5}\right)$ pair and a right-open $\left(\phi_{\mid 17}, \psi_{\mid 19}\right)$ one. The lhs of the comparison operator is evaluated using the values of counters $a_{\phi, \psi}, b_{\phi, \psi}$, and $h_{\phi, \psi}$ at time instants 19 and 5 (enclosed in a triangle in the figure ); its value is $\frac{5}{1}=5$. Now

\footnotetext{
2“if $A$ then $B$ else $C$ " can be written as $(A \wedge B) \vee(\neg A \wedge C)$
} 


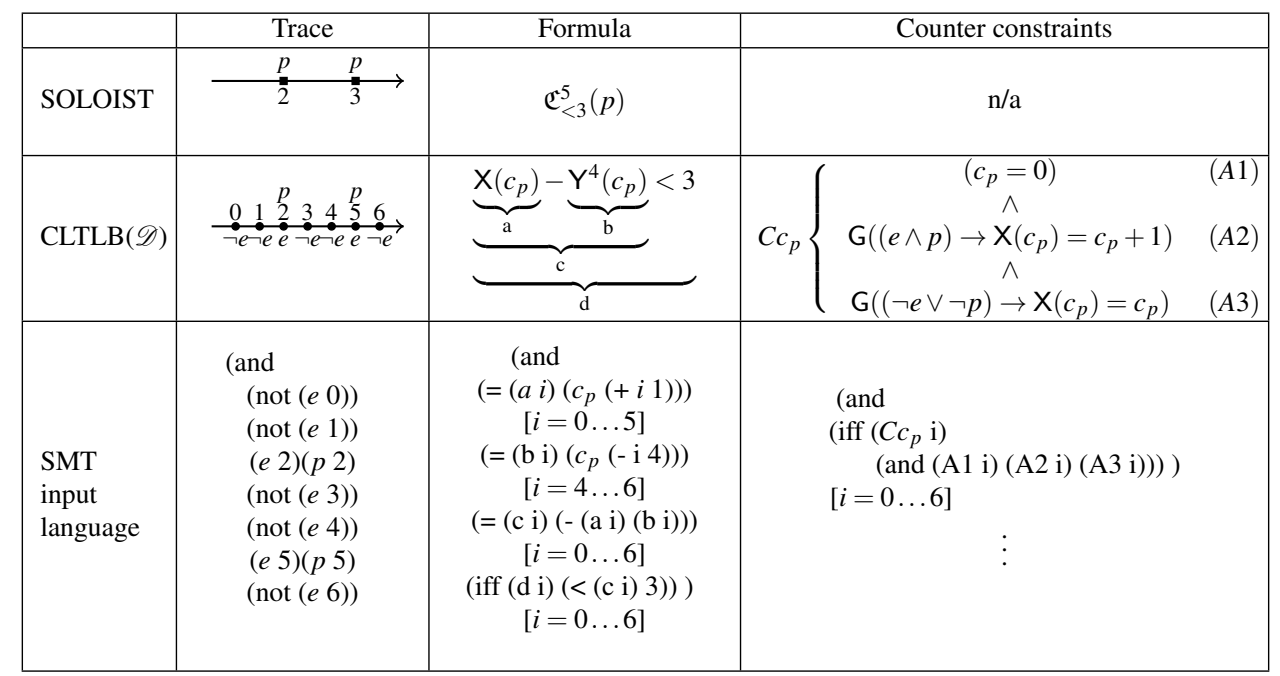

Figure 6: Example of the translation from SOLOIST to $\operatorname{CLTLB}(\mathscr{D})$ and then to the input language of the SMT solver

consider the formula $\mathfrak{D}_{\bowtie n}^{12}(\phi, \psi)$. When evaluated at time instant 15 , it has a left-open pair $\left(\phi_{\mid 2}, \psi_{\mid 5}\right)$. The values of the counters $a_{\phi, \psi}, b_{\phi, \psi}$, and $h_{\phi, \psi}$ considered to compute the lhs of the comparison operator are those at time instants 16 and 4 (enclosed in a square in the figure); the lhs evaluates to $\frac{5}{1}=5$. If the same formula is evaluated at time instant 18 , we find only a right-open pair $\left(\phi_{\mid 17}, \psi_{\mid 19}\right)$. The lhs of the comparison operator is evaluated using the value of counters $a_{\phi, \psi}$ and $h_{\phi, \psi}$ considered at time instants 19 and 7 (enclosed in a hexagon in the figure); its value is $\frac{5}{1}=5$.

\subsection{Complexity of the translation}

The translation function $\rho$, for the atomic propositions, the temporal modalities and all the aggregate ones, introduces a fixed-length formula; notice that subformulae occurring in aggregate modalities are restricted to be atomic. In the worst case, our translation is linear in the size of the input formula. We remark that we use a direct encoding of the exponent $K$ in formulae of the form $\mathrm{Y}^{K}$ or $\mathrm{X}^{K}$, both in the case of arithmetical temporal terms and of boolean formulae. The direct encoding of the exponent allows us to avoid expanding it into nested $Y$ or $\mathrm{X}$ formulae.

\section{Implementation}

The translation described in the previous section has been implemented in a tool [22]; this tool acts as a front-end for translating SOLOIST formulae into the input format of the ZOT verification toolset [26]. ZOT supports satisfiability checking of CLTLB $\mathscr{D})$ formulae by means of SMT solvers. A plugin-based architecture makes it easy to extend ZOT to support more expressive languages using $\operatorname{CLTLB}(\mathscr{D})$ as a core, and to output code for the different dialects of various SMT solvers. We implemented the support for SOLOIST as a ZOT plugin written in Common Lisp.

We now give a rundown of the translation steps applied to an example, to provide a glimpse of the implementation of our SMT-based trace checking algorithm. These steps and 
the example are also sketched in Fig. 6 where: the top row shows (a fragment of) the example input trace and the SOLOIST formula to verify on the trace; the middle row shows how the input trace is transformed from timed $\omega$-word to $\omega$-word, the translation of the input formula and the definition of the counter constraints as described in Sect.3, the bottom row shows how the trace, the input formula, and the counter constraints are translated into the input language of the SMT solver.

Let us consider the problem of performing trace checking of the formula $\phi \equiv \mathfrak{C}_{<3}^{5}(p)$ over the trace $H$ of length 7 depicted in Fig. 6, the formula is evaluated at time instant 5. As described in Sect. 3.1, our plugin translates the SOLOIST formula $\phi$ into CLTLB( $\mathscr{D})$ as $\rho(\phi) \equiv \mathrm{X}\left(c_{p}\right)-\mathrm{Y}^{4}\left(c_{p}\right)<3$, where $c_{p}$ is a counter. The behavior of this counter is constrained by the conjunction of axioms A $\mathrm{A}$. and $\mathrm{A} 3$, defined as $\mathscr{C}_{c_{p}} \equiv\left(c_{p}=0\right) \wedge \mathrm{G}((e \wedge p) \rightarrow$ $\left.\mathrm{X}\left(c_{p}\right)=c_{p}+1\right) \wedge \mathrm{G}\left((\neg e \vee \neg p) \rightarrow \mathrm{X}\left(c_{p}\right)=c_{p}\right)$. The next step is to invoke ZOT to translate the input formula and the counter constraints into the input language of the SMT solver. First, ZOT parses the formula and assigns a special proposition to each sub-formula in the input formula; similarly, it also assigns an arithmetic proposition to each arithmetical temporal term in the input formula. For example, as shown in Fig. 6, the arithmetic propositions $a$ and $b$ correspond, respectively, to the arithmetical temporal terms $\mathrm{X}\left(c_{p}\right)$ and $\mathrm{Y}^{4}\left(c_{p}\right) ; c$ is an arithmetical proposition holding the value of the $\mathrm{X}\left(c_{p}\right)-\mathrm{Y}^{4}\left(c_{p}\right)$ arithmetic temporal term; proposition $d$ corresponds to the entire input formula. The values of these auxiliary propositions are defined in each time instant $i=0 \ldots 6$, according to their semantics. The trace $H$ is also encoded in the input language of the SMT solver and provided to it as an assumption. The SMT solver is then fed with the translation, performed by ZOT, of the CLTLB $(\mathscr{D})$ formula $\neg\left(\mathrm{X}^{5}(\rho(\phi))\right) \wedge \mathscr{C}_{c_{p}}$. Notice that the formula $\phi$ is negated; hence, it is satisfied by trace $H$ if the SMT solver returns unsat. The exponent 5 in the term $X^{5}(\rho(\phi))$ is determined by the evaluation of the formula fixed at time instant 5 . The details of the translation from $\operatorname{CLTLB}(\mathscr{D})$ to the input language of the SMT solver (as sketched in the bottom row of Fig. 6 have been omitted since they are out of the scope of this work; for them, we refer the reader to [26].

\section{Evaluation}

We evaluated the effectiveness of our approach by investigating the following research questions:

- RQ1: How does the proposed approach scale with respect to the various parameters (e.g., the length of the trace, the length of the time window K) involved in SOLOIST trace checking? (Sect. 5.1)

- RQ2: How does the proposed trace checking procedure for SOLOIST based on CLTLB(D) compare with the procedure based on QF-EUFIDL [7]? (Sect.5.2)

- RQ3: Can the proposed trace checking procedure, based on CLTLB(D), handle traces more efficiently than the procedure used in our first implementation [9] 21], based on LTL? (Sect. 5.3)

- RQ4: Can the proposed trace checking procedure be applied in a realistic setting? (Sect.5.4)

Since there is no consolidated benchmark for service-based applications (for which SOLOIST was tailored), we decided to evaluate our approach using synthesized traces. These traces were obtained using the Process Log Generator (PLG) tool [11] on a model of the running example from Sect. 2.2. This model was defined by specifying the workflow structure, the duration 
of each synchronous invoke activity, the branching probabilities, and the error rates. Other activities (e.g., receive) were given 0 as duration; branching was used to create loops and simulate the behavior of the pick activity. The PLG tool is able to synthesize logs of process invocations from its input model. For each run of the trace checker, we recorded the memory usage, the translation time, and the SMT verification time. The evaluation was performed on a PC equipped with a 2.0GHz Intel Core i7-2630QM processor, running GNU/Linux Ubuntu 12.10 64bit, with 2GB RAM allocated for the verification tool. We used the Z3 [14] SMT solver v. 4.3.1.

\subsection{RQ1: Scalability of the approach}

To investigate RQ1, we considered the following parameters:

Trace length. It represents the length of the synthesized trace and the bound given to the SMT solver. The length of each synthesized trace depends on the duration of the activities invoking an external service as well as on the branching probabilities of the loop(s) in the process.

Length of the time window. It is used in the aggregate modalities; it corresponds to the $K$ parameter.

Bound of the comparison operator. It is used in the aggregate modalities; it corresponds to the $n$ parameter.

We present only the results of the evaluation done for the $\mathfrak{C}$ and $\mathfrak{D}$ modalities, since they are the keystones of the translation. We synthesized 20000 different traces, of variable length between 100 and 2000. We checked the following properties on them: $\mathfrak{C}_{>30}^{100}(p)$, and $\mathfrak{D}_{>30}^{100}(p, q)$, with propositions $p$ and $q$ corresponding to the start and end events of a service invocation of the process. The results of executing trace checking for each of these two properties on the synthesized traces are shown in Fig. $8 \mathrm{c}$ and $8 \mathrm{~d}$, in each row, the left plot shows the time for the translation and the one taken by the SMT solver, while the right plot shows the memory usage. Each point in the plot represents an average value of 10 trace check runs on traces of the same length. The plots provide an intuition of the growth rate of the resources usage with respect to the length of the input trace. The memory usage for the respective properties yields a very similar plot; we omitted it for space reasons. The memory dedicated for the evaluation of properties with the $\mathfrak{C}$ modality was exhausted at 2200 time instances, requiring $2.1 \mathrm{~GB}$ of memory and 40 seconds to solve. For the evaluation of the properties with the $\mathfrak{D}$ modality, the maximum number of time instances manageable before exhausting the preset memory limit was 2000 . The lower value with respect to the $\mathfrak{C}$ modality is due to the linear multivariate constraints introduced in the translation of the $\mathfrak{D}$ modality; these constraints are harder to solve than the univariate one used for the $\mathfrak{C}$ modality.

As for the scalability with respect to the other parameters, namely the length of the time window $K$ and the bound of the comparison operator $n$, we notice that they do not affect the resource usage, and only introduce some non-deterministic noise in the SMT solver time. This can be seen in Fig. 8e, which shows the time usage with respect to the variation of each of these two parameters when checking formulae over a synthesized trace of length fixed to 1000; the left plot refers to checking formula $\mathfrak{C}_{>5}^{K}(p)$, while the right one refers to checking formula $\mathfrak{C}_{>n}^{1000}(p)$. We omit the corresponding plots for the memory usage, since it is constant for any value of $K$ and $n$ in the formulae.

\subsection{RQ2: Comparison with the QF-EUFIDL-based encoding}

To investigate RQ2, we compared our approach with previous work for trace checking of SOLOIST [7]. This trace checking procedure is based on an encoding of SOLOIST proper- 
(a) Comparison between LTL and $\operatorname{CLTLB}(\mathscr{D})$
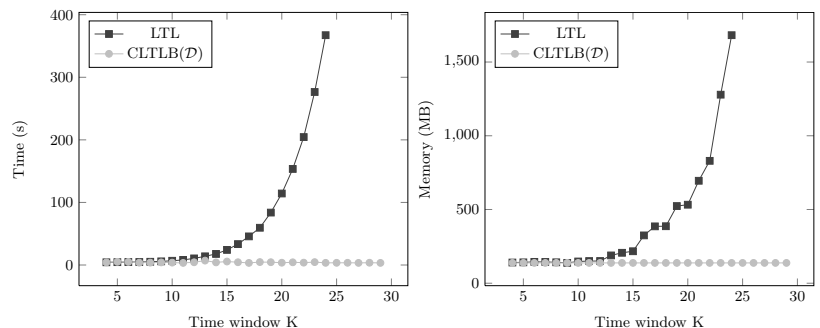

(b) Comparison between QFEUFIDL and $\operatorname{CLTLB}(\mathscr{D})$ over traces with $100 \%, 50 \%, 33 \%$, $25 \%, 20 \%, 16.6 \%$, and $14.3 \%$ sparseness
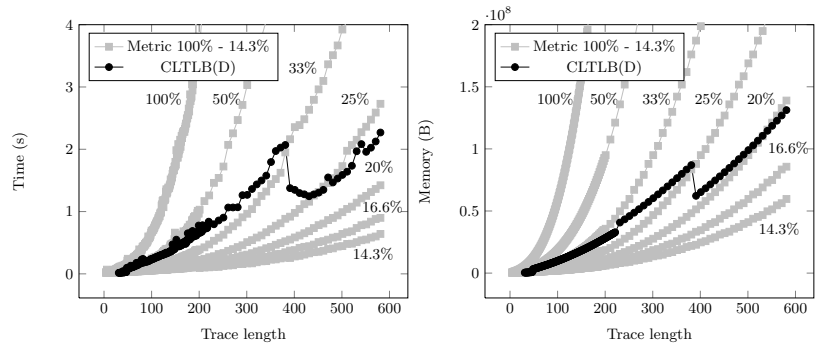

(c) Time and memory scalability of $\mathfrak{C}$ modality with respect to trace length $H$
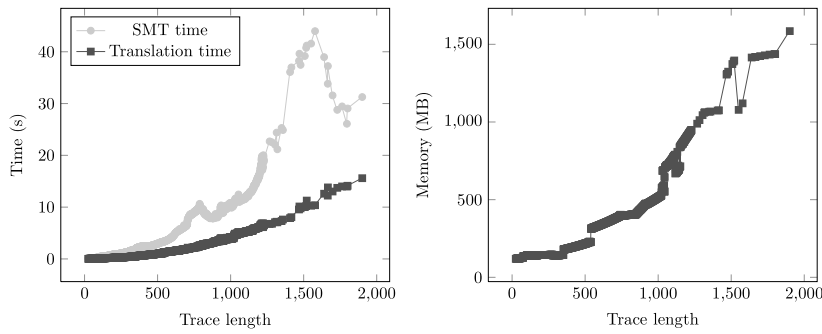

(d) Time and memory scalability of $\mathfrak{D}$ modality with respect to trace length $H$
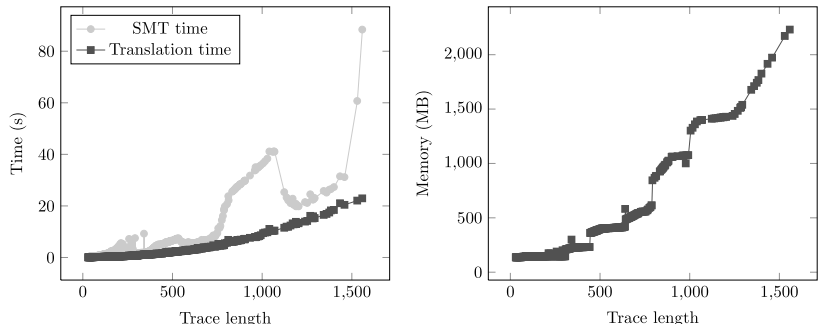

(e) Time scalability of $\mathfrak{C}$ modality with respect to time window $K$ (left) and the bound $n$ (right)
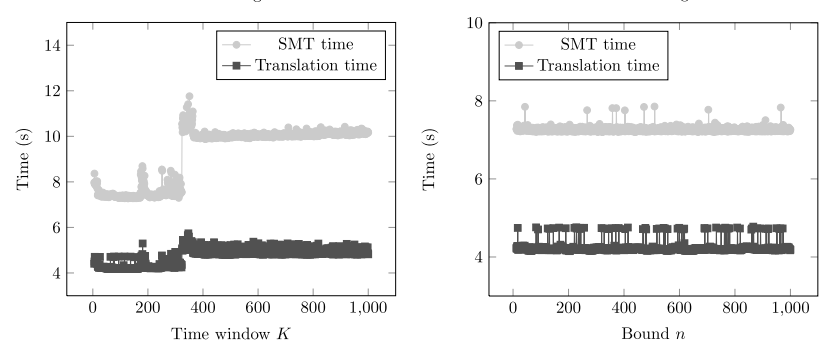

Figure 8: Evaluation of SOLOIST-based trace checking 
Table 1: Evaluation data of the running example

\begin{tabular}{cccccc}
\hline Property & Outcome & $\begin{array}{c}\text { ZOT } \\
\text { time (s) }\end{array}$ & $\begin{array}{c}\text { SMT } \\
\text { time (s) }\end{array}$ & $\begin{array}{c}\text { Total } \\
\text { time (s) }\end{array}$ & $\begin{array}{c}\text { Memory } \\
\text { (MB) }\end{array}$ \\
\hline & & \multicolumn{5}{c}{ (mean/standard deviation) } \\
\hline$(\mathrm{QP} 1)$ & true & $10.97 / 0.62$ & $19.70 / 1.55$ & $30.27 / 1.60$ & $931.0 / 0$ \\
$(\mathrm{QP} 2)$ & false & $20.77 / 1.37$ & $107.30 / 2.69$ & $125.50 / 2.29$ & $1261.7 / 118.24$ \\
$(\mathrm{QP} 3)$ & true & $10.56 / 0.46$ & $31.89 / 1.74$ & $42.08 / 1.73$ & $793.0 / 0$ \\
$(\neg \mathrm{QP} 1)$ & false & $11.59 / 0.75$ & $28.00 / 1.67$ & $38.94 / 1.73$ & $932.0 / 0$ \\
$(\neg \mathrm{QP} 2)$ & true & $20.74 / 0.69$ & $324.80 / 159.1$ & $343.00 / 158.2$ & $1210.8 / 80.47$ \\
$(\neg \mathrm{QP} 3)$ & false & $11.10 / 0.53$ & $31.37 / 0.55$ & $41.97 / 0.70$ & $942.0 / 0$ \\
\hline
\end{tabular}

ties into formulae of QF-EUFIDL, the theory of quantifier-free integer difference logic with uninterpreted function and predicate symbols. This encoding was tailored for sparse traces, i.e., traces in which the number of time instants when events occur is very low with respect to the length of the trace.

The comparison focuses on how the two approaches deal with traces of various sparseness degrees, where sparseness is defined as the ratio between the number of time instants in the trace where events occur and the total time the trace spans over. We compared the performance of the two approaches by classifying the generated traces into seven groups with $100 \%, 50 \%$, $33 \%, 25 \%, 20 \%, 16.6 \%$, and $14.3 \%$ of sparseness, respectively. We reevaluated the approach from [7] on traces from each group and compared time and memory requirements of both approaches. As shown in Fig. 8b, the approach presented in this paper is more efficient when the degree of sparseness of input traces is $25 \%$ or higher. The black line in the plot shows the performance of our approach, while the seven gray lines show our reevaluation of the approach based on QF-EUFIDL, applied to traces from the seven groups aforementioned.

\subsection{RQ3: Comparison with the LTL-based encoding}

To address RQ1, we synthesized a sample history trace of length 30 containing occurrences of an event $p$, and considered the SOLOIST formula $\mathfrak{C}_{>2}^{K}(p)$, which checks whether there have been more than two occurrences of the event $p$ within the last $K$ time units. We varied the length of the time window $K$ progressively from 2 to 30; the formula was always evaluated at the last time instant of the trace. Figure $8 \mathrm{a}$ shows the time and memory usage for the two translations. The results show that the length of the formula resulting from the translation into LTL depends on the size of the time window $K$. The translation from [9] was inefficient and produced a large encoding that resulted in a considerable increase in time and memory usage; our encoding addresses these issues, paving the way for a more efficient trace checking.

\subsection{RQ4: Application to a realistic example}

Here we report on the use of trace checking to assess whether the SOLOIST properties defined in Sect. 2.2 hold for the executions of the business process described in the running example. We performed the checks on 10 traces of length 1000 that we picked randomly from the set of synthesized traces. We checked the three properties as well as their negations; the average time and memory usage, as well as their standard deviation, are reported in table 11: these results show the practical feasibility of our approach. 


\section{Related Work}

This work lies in the wider area of research on verification of SBAs; we refer the reader to various surveys [10, 27, 12, 2], illustrating approaches both for design-time and for run-time verification of functional and QoS properties. In the rest of this section we focus on existing work on trace checking and verification of quantitative properties specified in languages similar to SOLOIST. For a detailed discussion on SOLOIST and related specification languages see [9].

Finkbeiner et al. [18] describe an approach to collect statistics over run-time executions. They extend LTL to return values from a trace and use them to compute aggregate properties of the trace. However, the specification language they use to describe the statistics to collect provides only limited support for timing information. For example, compared to SOLOIST, it cannot express properties on a certain subset of an execution trace. Furthermore, their evaluation algorithm relies on the formalism of algebraic alternating automata. These automata are manually built from the specification; thus making frequent changes to the property errorprone.

In reference [4] authors define an extension of metric first-order temporal logic (MFOTL) which supports aggregation. This language is very similar to SOLOIST with a general definition that supports any aggregate operator that can be defined as a mapping from multisets to $\mathbb{Q} \cup\{\perp\}$. The language can express aggregate properties over the values of the parameters of relations, while SOLOIST expresses aggregate properties on the occurrences of relations in the temporal first-order structure.

The trace checking approach presented in [3] exploits a Map-Reduce framework to validate properties of traces written in LTL. This work mainly focuses on recasting the trace checking problem into a Map-Reduce framework, by distributing (sub)trace validation tasks over many parallel sites.

In reference [5], authors introduce a specification language $P T L T L^{F O}$ (past time linear temporal logic with first-order (guarded) quantifiers) with a counting quantifier. It is used for expressing policies that can categorize the behavioral patterns of a user based on its transaction history. The counting quantifier counts the occurrences of an event from the beginning of the trace until the position of evaluation. The difference with the $\mathfrak{C}$ modality of SOLOIST is that there is no timing information: this means one cannot specify the exact part of the trace the modality should consider.

In reference [13], de Alfaro proposes pTL and pTL* as probabilistic extensions of CTL and CTL $^{*}$. These new languages include a new modality $\mathfrak{D}$ that expresses the bound on the average time between events. This is achieved by using an instrumentation clock that keeps track of the elapsed time from the beginning of the computation until the first occurrence of a specified event. To this end, the extended pTL formulae are evaluated on an instrumented timed probabilistic Markov decision process. Notice that the $\mathfrak{D}$ modality used in [13] differs from the one we introduced here, since it computes the time passed before the first occurrence of an event, averaged over the different computations of the underlying Markov decision process.

\section{Conclusion and Future Work}

The interactions among the various services participating in a composite SBA and the provisioning of such services can be characterized by precise specification patterns [8]. The SOLOIST language was developed [9] to express these patterns, which involve aggregate operations on events occurring in a given time window. In this paper, we propose an SMT- 
based offline trace checking procedure for SOLOIST. This approach exploits a translation of SOLOIST into CLTLB $(\mathscr{D})$, a variant of linear temporal logic that supports counter variables. We assess the scalability of the approach with respect to the various parameters involved in SOLOIST trace checking, and we also compare it with previous work.

The use of SOLOIST in the context of practical verification activities is the goal of further on-going research and we intend to validate our proposal in realistic scenarios, in collaboration with industrial partners. After further improvements to the translation, we also plan to move from offline trace checking to run-time verification, integrating ZOT and the SOLOIST plugin into a run-time monitoring framework for SBAs.

\section{Acknowledgments}

This work has been partially supported by the National Research Fund, Luxembourg (FNR/P10/03). The authors wish to thank Marcello Bersani and Matteo Pradella for their precious help with ZOT.

\section{References}

[1] Luciano Baresi, Domenico Bianculli, Carlo Ghezzi, Sam Guinea, and Paola Spoletini. Validation of web service compositions. IET Softw., 1(6):219-232, 2007.

[2] Luciano Baresi and Elisabetta Di Nitto, editors. Test and Analysis of Web Services. Springer, 2007.

[3] Benjamin Barre, Mathieu Klein, Maxime Soucy-Boivin, Pierre-Antoine Ollivier, and Sylvain Hallé. MapReduce for Parallel Trace Validation of LTL Properties. In Proc. of $R V$ 2012, volume 7687 of LNCS, pages 184-198. Springer, 2013.

[4] David Basin, Felix Klaedtke, Srdjan Marinovic, and Eugen Zălinescu. Monitoring of temporal first-order properties with aggregations. In Proc. of $R V^{\prime} 13$, volume 8174 of LNCS, pages 40-58. Springer, 2013.

[5] Andreas Bauer, Rajeev Goré, and Alwen Tiu. A first-order policy language for historybased transaction monitoring. In Proc. of ICTAC '09, volume 5684 of LNCS, pages 96-111. Springer, 2009.

[6] Marcello M. Bersani, Achille Frigeri, Angelo Morzenti, Matteo Pradella, Matteo Rossi, and Pierluigi San Pietro. Constraint ltl satisfiability checking without automata. CoRR, abs/1205.0946, 2012.

[7] Marcello Maria Bersani, Domenico Bianculli, Carlo Ghezzi, Srđan Krstić, and Pierluigi San Pietro. SMT-based checking of SOLOIST over sparse traces. In Proceedings of FASE 2014, volume 8411, pages 276-290. Springer, April 2014.

[8] Domenico Bianculli, Carlo Ghezzi, Cesare Pautasso, and Patrick Senti. Specification patterns from research to industry: a case study in service-based applications. In Proceedings of ICSE 2012, pages 968-976. IEEE, June 2012.

[9] Domenico Bianculli, Carlo Ghezzi, and Pierluigi San Pietro. The tale of SOLOIST: a specification language for service compositions interactions. In Proceedings of FACS 2012, volume 7684, pages 55-72. Springer, September 2012. 
[10] Mustafa Bozkurt, Mark Harman, and Youssef Hassoun. Testing \& verification in serviceoriented architecture: A survey. Softw. Test. Verif. Reliab., 2012.

[11] Andrea Burattin and Alessandro Sperduti. PLG: A framework for the generation of business process models and their execution logs. In Business Process Management Workshops, volume 66 of LNBIP, pages 214-219. Springer, 2011.

[12] Gerardo Canfora and Massimiliano Di Penta. Service oriented architectures testing: a survey. In ISSSE 2006-2008, volume 5413 of LNCS, pages 78-105. Springer, 2009.

[13] Luca de Alfaro. Temporal logics for the specification of performance and reliability. In Proc. of STACS'97, volume 1200 of LNCS, pages 165-176. Springer, 1997.

[14] Leonardo Mendonça de Moura and Nikolaj Bjørner. Z3: An Efficient SMT Solver. In Proc. of TACAS 2008, volume 4963 of LNCS, pages 337-340. Springer, 2008.

[15] Stéphane Demri and Deepak D'Souza. An automata-theoretic approach to constraint LTL. Inf. Comput., 205(3):380-415, 2007.

[16] Matthew B. Dwyer, George S. Avrunin, and James C. Corbett. Property specification patterns for finite-state verification. In Proc. of FMSP '98, pages 7-15. ACM, 1998.

[17] Miguel Felder and Angelo Morzenti. Validating real-time systems by history-checking TRIO specifications. ACM Trans. Softw. Eng. Methodol., 3(4):308-339, October 1994.

[18] Bernd Finkbeiner, Sriram Sankaranarayanan, and HennyB. Sipma. Collecting statistics over runtime executions. Formal Methods in System Design, 27:253-274, 2005.

[19] Nicolai Josuttis. SOA in Practice: The Art of Distributed System Design. O'Reilly Media, Inc., 2007.

[20] Sascha Konrad and Betty H. C. Cheng. Real-time specification patterns. In Proc. of ICSE '05, pages 372-381. ACM, 2005.

[21] Srđan Krstić. Verification of quantitative properties of service-based applications, December 2012.

[22] Srđan Krstić. SOLOIST Translator. https://bitbucket.org/krle/ soloist-translator 2013.

[23] Orna Lichtenstein, Amir Pnueli, and Lenore Zuck. The glory of the past. In Proc. of Logics of Programs, volume 193 of LNCS, pages 196-218. Springer, 1985.

[24] Aouatef Mrad, Samatar Ahmed, Sylvain Hallé, and Èric Beaudet. Babeltrace: A collection of transducers for trace validation. In Proc. of RV 2012, volume 7687 of LNCS, pages 126-130. Springer, 2013.

[25] Matteo Pradella, Angelo Morzenti, and Pierluigi San Pietro. The symmetry of the past and of the future: bi-infinite time in the verification of temporal properties. In Proc. of ESEC-FSE' '07, pages 312-320. ACM, 2007.

[26] Matteo Pradella, Angelo Morzenti, and Pierluigi San Pietro. Bounded satisfiability checking of metric temporal logic specifications. ACM Trans. Softw. Eng. Methodol., 22(3):20:1-20:54, July 2013.

[27] Gwen Salaün. Analysis and verification of service interaction protocols - a brief survey. In Proc. of TAV-WEB 2010, volume 35 of EPTCS, pages 75-86, 2010. 